\title{
Sustained analgesic effect of clonidine co-polymer depot in a porcine incisional pain model
}

This article was published in the following Dove Press journal: Journal of Pain Research

\section{Jared T Wilsey \\ Julie H Block}

Medtronic Spine Division, Memphis, TN, USA

Correspondence: Jared T Wilsey Medtronic Spinal and Biologics Division, 2600 Sofamor Danek Drive, Memphis,

TN 38I32, USA

$\mathrm{Tel}+19013992630$

Fax +19013992560

Email jared.t.wilsey@medtronic.com
Background: Previous research suggests that the $\alpha_{2}$ adrenergic agonist clonidine, a centrally acting analgesic and antihypertensive, may also have direct effects on peripheral pain generators. However, aqueous injections are limited by rapid systemic absorption leading to off target effects and a brief analgesic duration of action.

Purpose: The aim of this study was to examine the efficacy of a sustained-release clonidine depot, placed in the wound bed, in a pig incisional pain model.

Methods: The depot was a $15 \mathrm{~mm} \times 5 \mathrm{~mm} \times 0.3 \mathrm{~mm}$ poly(lactide-co-caprolactone) polymer film containing 3\% (w/w) clonidine $\mathrm{HCl}$ (MDT3). Fifty-two young adult mix Landrace pigs $(9-11 \mathrm{~kg})$ were divided into seven groups. All subjects received a $6 \mathrm{~cm}$, full-thickness, linear incision into the left lateral flank. Group 1 served as a Sham control group (Sham, $n=8$ ). Group 2 received three placebo strips $(\mathrm{PBO}, \mathrm{n}=8$ ), placed end-to-end in the subcutaneous wound bed before wound closure. Group 3 received one MDT3 and two PBO $(n=8)$, Group 4 received two MDT3 and one PBO ( $n=8)$, and Group 5 received three MDT3 ( $n=8)$. Positive control groups received peri-incisional injections of bupivacaine solution (Group 6, $30 \mathrm{mg}$ /day bupivacaine, $\mathrm{n}=8$ ) or clonidine solution (Group 7, $225 \mu \mathrm{g}$ /day, $\mathrm{n}=4$ ).

Results: The surgical procedure was associated with significant peri-incisional tactile allodynia. There was a dose-dependent effect of MDT3 in partially reversing the peri-incisional tactile allodynia, with maximum pain relief relative to Sham at 72 hours. Daily injections of bupivacaine (30 mg), but not clonidine (up to $225 \mu \mathrm{g}$ ), completely reversed allodynia within 48 hours. There was a statistically significant correlation between the dose of MDT3 and cumulative withdrawal threshold from 4 hours through the conclusion of the study on day 7.

Conclusion: These data suggest that a sustained-release clonidine depot may be a viable nonopioid, nonamide anesthetic therapy for the treatment of acute postsurgical nociceptive sensitization. Keywords: amide anesthetic, bio-erodible polymer, peripherally acting analgesic, imidazoline, porcine model, postoperative pain, regional anesthesia, sustained release

\section{Introduction}

There is an increasing emphasis on directly addressing local pain generators following surgery in an effort to reduce systemic exposure to narcotics and other medications. ${ }^{1-5}$ Bupivacaine and related local anesthetics have been encapsulated in various depot formulations as potential treatments for postoperative pain. The primary limitation to such products has been a relatively short duration of action, conferring little temporal advantage over regional blocks and local infiltration procedures with standard solution for injection. For example, a Phase III bunionectomy trial with a bupivacaine liposome formulation demonstrated minimal analgesia $>24$ hours. ${ }^{6}$ For orthopedic 
surgical procedures on the extremities or spine, the majority of patients experience moderate-to-severe pain for at least 96 hours. $^{7}$ Another significant issue with amino-amide anesthetics is local tissue toxicity in the therapeutic dosage range, with muscle and cartilaginous tissue appearing to be particularly vulnerable. ${ }^{8}$ Because local tissue toxicity is both time and concentration dependent, ${ }^{9}$ this class of medications may be poorly suited for $>48$ hours sustained-release formulations. To overcome these limitations, a biodegradable clonidine-eluting implant with a sustained drug release profile was developed. This investigational product, MDT3, is in the form of a thin, flexible $15 \mathrm{~mm} \times 5 \mathrm{~mm} \times 0.3 \mathrm{~mm}$ poly(lactide-co-caprolactone) polymer film containing $3 \%$ (w/w) clonidine $\mathrm{HCl}$ (0.77 mg).

An early screening program included the evaluation of $>30$ drugs and biologics, including analgesics, glucocorticoids, and nonsteroidal anti-inflammatory agents. Clonidine was chosen for this program primarily because it was superior to other candidates in nonclinical assessments of local tissue tolerance as well summed efficacy, the latter of which was the focus of this investigation. Currently approved as an epidural analgesic for intractable cancer pain, accumulating evidence demonstrates distinct peripheral-mediated mechanisms for pain relief. Anti-inflammatory effects have been demonstrated in humans ${ }^{10,11}$ and numerous animal models. ${ }^{12-14}$ Local, perioperative administration of $150 \mu \mathrm{g}$ clonidine $\mathrm{HCl}$ has been shown to reduce the pain associated with orthopedic surgery. This dose of clonidine was effective when injected into the intra-articular space during arthroscopic knee surgery ${ }^{15-17}$ or when injected into neighboring muscle tissue during arthroscopic shoulder surgery. ${ }^{18}$ Consistent with a local mechanism of action, subcutaneous administration of the same dose was ineffective in both studies.

The efficacy of direct application of clonidine into the surgical site is transient, typically dissipating by 24 hours postadministration in referenced clinical studies. The concept for MDT3 was to develop a method for sustaining pharmacologically active concentrations of clonidine in healing tissue for several days. In vivo and in vitro release studies confirmed at least 5 days of sustained release from the strip. Local tissue distribution studies found average local tissue concentrations of $\sim 100 \mathrm{ng} / \mathrm{g}$ of tissue following intermuscular (IM) placement of two strips for at least 7 days (data not shown). While this is an order of magnitude above the therapeutic range for CSF concentrations associated with analgesia using Duraclon ${ }^{\circledR}$ (a $100 \mu \mathrm{g} / \mathrm{mL}$ preparation of clonidine $\mathrm{HCl}$ administered into epidural space for intractable cancer pain), the threshold tissue concentration required for peripherally mediated (local) analgesia is uncertain. Here, we describe the efficacy of MDT3 in a porcine incisional pain model. This model was selected for physiological similarities in dermal tissue to man because it appears to be associated with minimal spontaneous pain, with no changes in food consumption, weight gain, or other signs of pain behavior when unperturbed. ${ }^{19}$ Although substantial historical data are available for various rodent-induced pain models including the Brennan et al's ${ }^{20}$ incisional pain model, such a model could not physically accommodate the test article as sized and may not tolerate systemic (off target) absorption. An earlier validation study demonstrated significant peri-incisional tactile allodynia that was responsive to systemic analgesics or local anesthetics. ${ }^{19}$ These characteristics, and especially the lack of obvious spontaneous pain, allowed the testing of the acute analgesic effects of MDT3 monotherapy. If a model is associated with obvious signs of spontaneous pain or pain at rest, it is generally considered necessary from an ethical perspective to administer a systemic analgesic (eg, buprenorphine) in a multiday study that, in turn, could prevent the assessment of test article as a standalone analgesic, particularly if the onset of action proves to be delayed.

\section{Methods}

Fifty-two young mix Landrace male pigs, weighing $10.70 \pm 0.78 \mathrm{~kg}$ at study initiation, were acquired for this study (purpose bred for research from Kibbutz Lahav, Israel). Animals were drug and test naive and considered in good health by the attending veterinarian. Animals were randomly divided into seven groups ( ix groups of $n=8$ and one group of $n=4$ [clonidine solution control]) as listed later (Table 1). Sample size was chosen to achieve an $80 \%$ power to detect a $25 \%$ between-group difference in mechanical withdrawal threshold at 72 hours and assume a pooled standard deviation of $\sim 20 \%$ (estimate of variance based on unpublished pilot data in the same model). Animals were provided with a commercial pig diet with ad libitum access to food and water. Purina ${ }^{\circledR}$ Dog Chow ${ }^{\circledR}$ was provided as a treat during acclimation to handlers

Table I Description of treatment groups

\begin{tabular}{llll}
\hline Group \# & $\mathbf{n}$ & Treatment group & $\begin{array}{l}\text { Treatment } \\
\text { (implant configuration) }\end{array}$ \\
\hline $\mathrm{I}$ & 8 & Sham & None \\
2 & 8 & Placebo & PBO; PBO; PBO \\
3 & 8 & I MDT3 & PBO; MDT3; PBO \\
4 & 8 & 2 MDT3 strip & MDT3; PBO; MDT3 \\
5 & 8 & 3 MDT3 strip & MDT3; MDT3; MDT3 \\
6 & 8 & Bupivacaine $(30 \mathrm{mg})$ & $333 \mu \mathrm{L} ; 333 \mu \mathrm{L} ; 333 \mu \mathrm{L}$ \\
7 & 4 & Clonidine $(225 \mu \mathrm{g})$ & $333 \mu \mathrm{L} ; 333 \mu \mathrm{L} ; 333 \mu \mathrm{L}$ \\
\hline
\end{tabular}

Abbreviations: PBO, placebo; MDT3, clonidine strips 
and then to distract animals during incisional pain testing. Animals were group housed in a $>10 \mathrm{~m}^{2}$ fenced pen within an open air-roofed shelter facility deemed appropriate for the temperate winter climate at the study site (Nes Ziona, Israel). Animals had continuous access to fresh water, except during the tactile allodynia testing described later. All procedures were approved by the MDBiosciences Institutional Animal Care and Use Committee (Nes Ziona, Israel) and adhere to the US National Research Council Guide for the Care and Use of Laboratory Animals (8th edition). Reporting in this article complies with ARRIVE guidelines (NC3R, London, UK).

\section{Test article}

Clonidine $\mathrm{HCl}$ was obtained from Societa' Italiana Medicinali Scandiccini (SIMS, Reggelllo, Italy) and milled by Micron Technologies (Lavern, PA, USA) prior to incorporation into copolymer. The micronized clonidine $\mathrm{HCl}$ was mixed with poly(DL-lactide-co-caprolactone) at $\sim 3 \%(\mathrm{w} / \mathrm{w})$ and melt extruded into a $0.3 \mathrm{~mm}$ width sheet that was cut into $\sim 15 \mathrm{~mm}$ $\times 5 \mathrm{~mm}$ strips. This process renders a bioabsorbable film not dissimilar in appearance or principle from oral breath strips, albeit with much slower hydrolytic degradation. Similar sized PBO, not containing clonidine, was also manufactured. Bupivacaine and clonidine solutions were made from $\mathrm{HCl}$ salt powders purchased from Sigma-Aldrich Co. (St Louis, MO, USA) (product numbers B5274 and C7897, respectively). Fully dissolved solutions were filtered $\left(0.22 \mu \mathrm{M}\right.$ Nalgene ${ }^{\circledR}$ syringe filter, Rochester, NY, USA) into sterile vials prior to injection.

\section{von Frey filament testing acclimation and baseline procedure}

During a 2-week acclimation period, animals were hand-fed Dog Chow $^{\circledR}$ twice daily for 15 minutes to simulate testing conditions. Three days prior and then again 1 day prior to surgery, animals were introduced to the von Frey apparatus (set of 20 von Frey filaments spanning 0.008-300 g) (Stoelting, Dale Wood, IL, USA). The last measurement taken at the planned surgical site on Day 1 serves as the baseline (day of surgery designated as "day 0"). The withdrawal threshold is the strength of filament required to induce a withdrawal response (moving or jerking away from the stimulus and/or vocalization consistent with a pain response).

\section{Surgical procedure}

Piglets were anesthetized with isoflurane $(0.5-5 \%)$ with $\mathrm{O}_{2}$ carrier (2-3 L/min), adjusted to maintain a surgical plane of anesthesia. This anesthesia was chosen to allow relatively rapid recovery for the assessment of primary endpoint beginning at 4 hours. A sterile cover was placed to surround the incisional area in the left lateral flank, and the skin was cleaned with Hibiclens ${ }^{\circledR}$ solution and $70 \%$ ethanol solution. A $6 \mathrm{~cm}$ long, full thickness skin incision, including the panniculus carnosus muscle, was made in each animal (Figure 1). All animals in Groups 2-5 received a total of three poly(lactide-co-caprolactone) strips. Animals in the placebo group (Group 2) received three PBO strips, placed end-toend with the length of the strip parallel to the incision, in the wound bed prior to closure. Animals in the $1 \times$ MDT3 group (Group 3) also received two PBO, with the active strip placed in the middle and the three strips similarly placed end-to-end along the length of the incision. Animals in the $2 \times$ MDT3 group (Group 4) also received one PBO, with the inactive PBO in the middle. Finally, animals in the $3 \times$ MDT3 group (Group 5) received the three active strips placed end-to-end in the wound bed. Active control groups (Group 6, bupivacaine; and Group 7, clonidine) did not receive test article during the surgical procedure. The positive control groups received daily $1 \mathrm{~mL}$ injections of either bupivacaine $(30 \mathrm{mg} / \mathrm{mL})$ or clonidine $(225 \mu \mathrm{g} / \mathrm{mL})$. These daily doses were divided into three $\sim 333 \mu \mathrm{L}$ subcutaneous injections via a $31 \mathrm{G}$ needle entering skin $\sim 1 \mathrm{~cm}$ medial to the midpoint, cranial end, and caudal end of the incision, with the bevel directed in an attempt to pool solution in the sub-incisional space. The sub-incisional route of administration for MDT3 was chosen consistent with the hypothesis that a clonidine depot could have a localized effect of peripheral pain generator(s). The completion of strip placement was recorded as time 0. Since Sham animals (Group 1) and positive control animals (Groups 6 and 7) did not receive test article or $\mathrm{PBO}$ prior to wound closure, the initiation of wound closure was recorded as time 0 in these
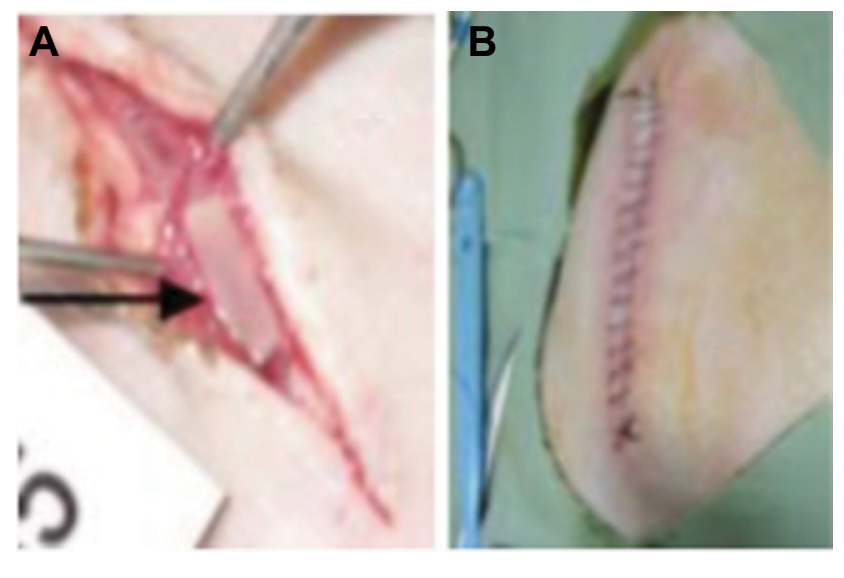

Figure I (A) Linear incision opening, showing placement of MDT3 mid-incision. A total of three strips were placed end-to-end in Groups 2-5 (MDT3, placebo, or a combination thereof). (B) The sutured incision postsurgery on day 0 . 
groups. The skin was closed using a sterile $3-0$ silk suture ( $\sim 12$ continuous stitches). Following surgery, the pigs were given a $0.5 \mathrm{~mL}$ injection of $10 \%$ marbocyl IM. The total time under anesthesia was $\sim 15$ minutes for each animal, during which time heart rate and blood oxygen saturation were monitored.

\section{Tactile withdrawal threshold assessments}

Following recovery from anesthesia on the day of surgery, the pain behavior threshold was measured by applying von Frey monofilaments adjacent to the wound. This first assessment was conducted 4 hours postoperative. The von Frey filaments were applied just lateral $(<2 \mathrm{~cm})$ to the mid-point and just in front of the cranial end of the incision using the Dixon up-down method. Each filament was applied a minimum of three times. Pain or "withdrawal" response was characterized by the animal reactively moving away from the stimulus by quickly stepping away and/or twisting the flank area. This testing procedure was repeated at 24 hours' intervals from the approximate time of dosing for 1-week postoperative. Both the tester and recorder were blinded as to the treatment of each animal.

\section{Spontaneous locomotor activity}

Spontaneous locomotor activity was quantified to indirectly assess sympatholytic, sedative effects of systemic clonidine. The open field consisted of a $270 \mathrm{~cm} \times 180 \mathrm{~cm}$ fenced rectangular grid, subdivided into $54^{2}\left(900 \mathrm{~cm}^{2}\right)$ cells (nine rows of six). Animals were acclimated to the open field test during the week prior to surgery, with data collection beginning the day prior to surgery (baseline). Tests were again conducted following tactile allodynia tests at $\sim 4,24$, and 48 hours postsurgery (capturing the projected period of peak systemic exposure). Animals were individually assessed by guiding them into the open field area from their pens, closing the gated fence surrounding the field and leading back to pens, and manually recording movement across cells for 2 minutes. A technician recorded movement on a map of the open field, marking a vertical line each time both front feet entered a cell. The number of cell boundary breaches during the 2-minute observation period was summed for each animal and for each test.

\section{In vitro drug release}

Single MDT3 strips were weighed and placed in $5 \mathrm{~mL}$ cryovials. A total of $4 \mathrm{~mL}$ of $1 \times$ PBS receiving buffer was added to each vial, with testing conducted in triplicate. The vials were then placed in a shaking water bath set to $37^{\circ} \mathrm{C}$ and $120 \mathrm{rpm}$. The samples were pulled from the water bath at the following time points: 4 hours, 1 day, 2 days, 3 days, 4 days, 7 days, 10 days, and 14 days. At each pull, the $4 \mathrm{~mL}$ of buffer was removed from each sample vial and replaced with $4 \mathrm{~mL}$ of fresh receiving buffer. The vials were then placed back into the shaker bath. An aliquot of the buffer that was removed from each sample was transferred into HPLC vials for analysis (mobile phase 75:25 $\mathrm{H}_{2} \mathrm{O}: \mathrm{CH}_{3} \mathrm{CN}$; flow rate $1.2 \mathrm{~mL} / \mathrm{min}$; column XBridge C8 $4.6 \times 150 \mathrm{~mm}, 5 \mu \mathrm{M}$; Waters, Milford, MA, USA). The concentration of clonidine $\mathrm{HCl}$ in each aliquot was used to calculate net drug release during each interval.

\section{Plasma clonidine}

In order to avoid potential noise introduced into the behavioral data as a result of the stress of frequent blood collection, blood draws were not completed in the 52 study animals. A cohort, $n=4$, of Landrace mixed pigs of similar age and body mass was administered a single subcutaneous MDT3 strip. Blood was drawn from an ear vein prior to placement and at 4 hours postadministration, 24 hours postadministration, and then daily through 1 week. Approximately $1 \mathrm{~mL}$ of blood was transferred to EDTA K2-coated tubes and centrifuged at $3000 \mathrm{rpm}$ for 10 minutes. Plasma was then transferred to cryovials and stored at $-70^{\circ} \mathrm{C}$. Samples were shipped to InVentiv Health Clinical (Quebec, QC, Canada) for analysis. Clonidine was extracted from plasma using a liquid-liquid extraction procedure and injected into a liquid chromatograph equipped with tandem mass spectrometry detector. Quantitation is by peak area ratio. A weighted (1/C2) linear regression standard curve spanning analytical range 20.0-2000 pg/mL $\left(R^{2}>0.98\right)$ was calculated using standards prepared by InVentiv and using the Thermo Electron Corporation Watson LIMS software Version 7 (Thermo Fisher Scientific, Waltham, MA, USA).

\section{Statistical analysis}

Data were analyzed using the GraphPad Prism ${ }^{\circledR}$ (Version 7.02) Software (GraphPad Software, Inc., La Jolla, CA, USA). Since withdrawal threshold scores were similar cranial to the incision and lateral to the mid-point, only the analyses of the mid-incision data are presented in this article. Withdrawal threshold data over time were analyzed by repeatedmeasures ANOVA and Dunnett's post hoc, comparing each treatment group with Sham at each time point. Locomotor activity and additional analyses are described in Figures 2-4. Average hourly rate of drug release was calculated from total drug release during each interval between buffer changes 


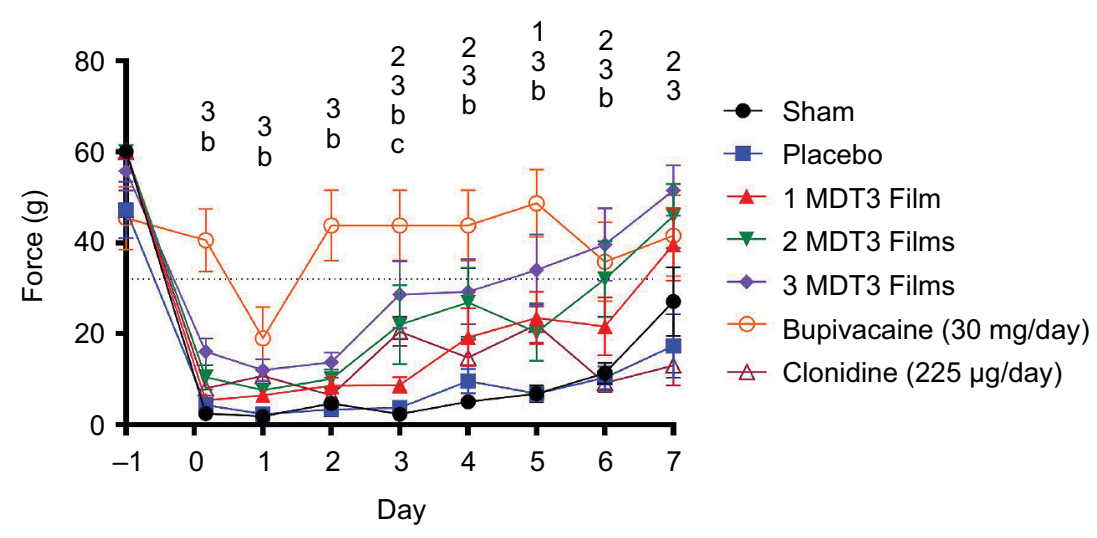

Figure 2 Mechanical withdrawal threshold expressed as a percent of baseline.

Notes: Data represent mean \pm SEM. Data were analyzed by repeated measures ANOVA. Both the time $(P<0.000 I, F=49.8)$ and the treatment $(P<0.000 I, F=2 I .5)$ main effects reached statistical significance, as did the interaction between main effects $(P<0.000 \mathrm{I}, F=2.45)$. As such, factors were further separated with each treatment group compared to Sham by Dunnett's multiple comparison test at each time point. ' $P<0.05$ for difference between one film and Sham at day 5 . ${ }^{2} P<0.05$ for difference between two films and Sham on days $3,4,6$ and 7 . ${ }^{3} P<0.05$ for difference between three films and Sham at 4 hours and days $I, 2,3,4,5,6$, and 7. ${ }^{b} P<0.05$ for difference between bupivacaine and Sham at 4 hours and days I, 2, 3, 4, 5, and 6. ${ }^{c} P<0.05$ for difference between clonidine $(225 \mathrm{mg} /$ day $)$ and Sham on day 3.

Abbreviation: MDT3, clonidine strips

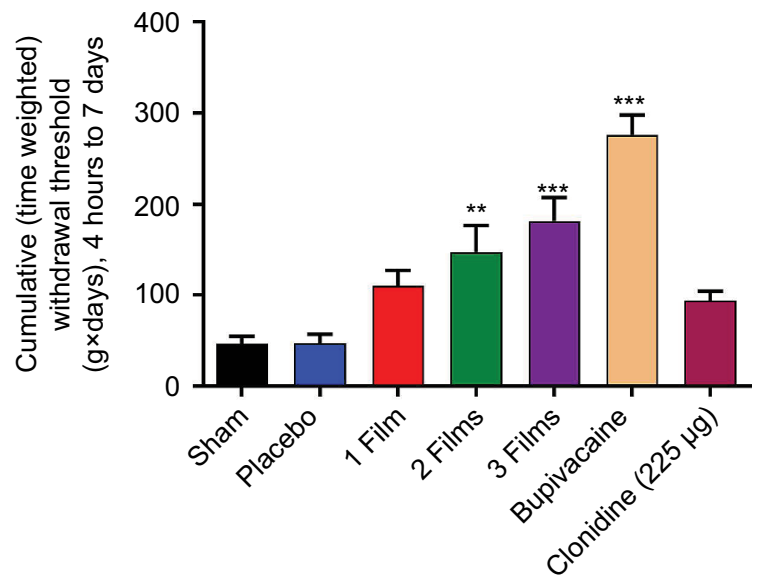

Figure 3 Cumulative (time weighted) withdrawal threshold, 4 hours to 7 days postsurgery (g days).

Notes: Data represent mean \pm SEM. Data were analyzed by ANOVA $(P<0.0001$, $F=15.82$ ) followed by Dunnett's multiple comparison test, comparing each group with Sham. ${ }^{* * P}<0.01$ for two strips versus Sham. ${ }^{* * *} P<0.000$ I for three strips versus Sham; $P<0.000$ I for bupivacaine versus Sham.

(Figure 5). Human equivalent dose (HED) was calculated by the following formula: HED = animal dose in $\mathrm{mg} / \mathrm{kg} \times$ (animal weight in $\mathrm{kg} / \mathrm{human}$ weight in $\mathrm{kg})^{1 / 3}$, with $60 \mathrm{~kg}$ used for human weight as described in Food and Drug Administration (US FDA) safety guidance. All data graphs were created in GraphPad Prism ${ }^{\circledR}$, with error bars representing standard error of the mean (Figures 2-4) or 95\% confidence limits (Figure 5).

\section{Results}

As expected, the surgical procedure was associated with significant peri-incisional tactile allodynia. In the Sham group, a $>95 \%$ reduction in mean withdrawal threshold was recorded at 4 hours (Figure 2) and remained $>80 \%$ below baseline through

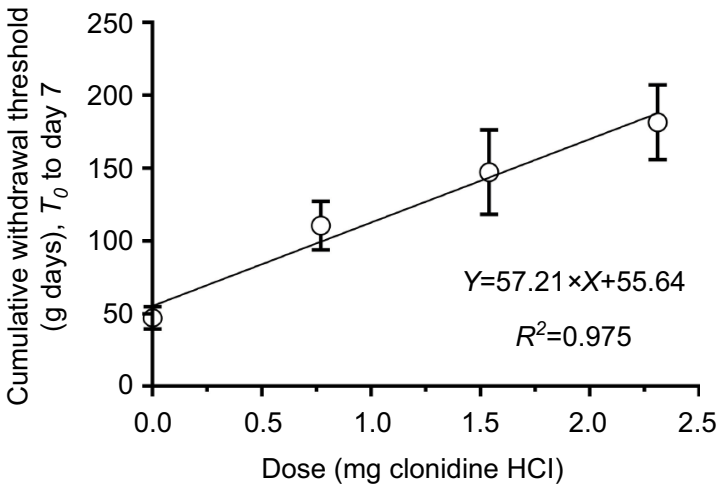

Figure 4 Linear regression of cumulative (time weighted) withdrawal threshold (4 hours to 7 days postsurgery [g days]) versus dose (line).

Notes: Data represent mean \pm SEM. The correlation between dose and cumulative efficacy reached significance (Pearson $r=0.988, P<0.01$ ). The best-fit linear regression equation is shown in standard $y=$ (slope) $x+y$ intercept format. Slope was significantly positive $(P<0.0$ l against null hypothesis slope $=0)$.

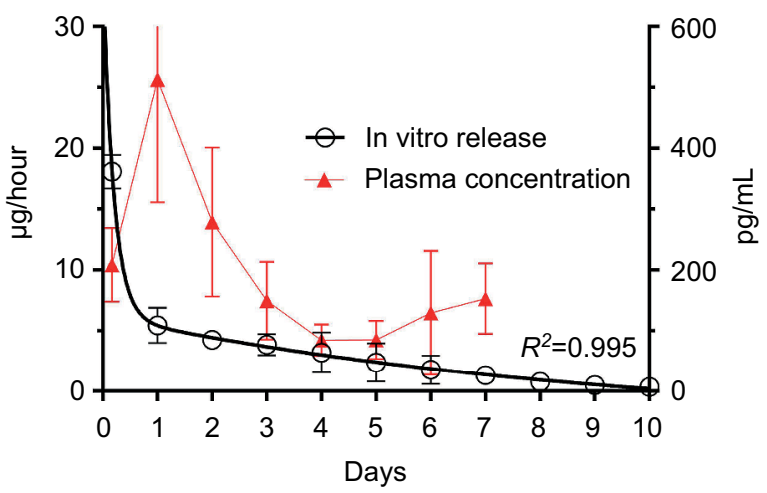

Figure 5 Average in vitro rate of drug release (micrograms clonidine $\mathrm{HCl}$ per hour) and plasma concentration following administration of a single clonidine film.

Notes: In vitro release data represent mean of three replicates $( \pm 95 \%$ confidence limits). The data are well described $\left(R^{2}>0.99\right)$ by a two-phase exponential decay model using least squares method with the following constraints: $K I>0, K 2>0$, and plateau $\geq 0$. $K I=0.1484 /$ hour $(T I / 2=4.7$ hours), $K 2=6.038 / \mathrm{hr} \quad(T I / 2=0.1 \mathrm{I}$ hours), plateau $=0$. Observed plasma $C_{\max }$ was $279 \pm 50.1 \mathrm{pg} / \mathrm{mL}$ at 24 hours, falling to $149 \pm 26 \mathrm{pg} / \mathrm{mL}$ at 72 hours. 
Day 5 , with recovery to $\sim 50 \%$ of baseline by Day 7 (Figure 2 ). PBO alone did not have a significant effect on the surgeryrelated tactile allodynia at any time point. There was a dosedependent effect of MDT3 in reversing surgery-related tactile allodynia. The effect of three MDT3 strips reached statistical significance by 4 hours, whereas the effect of two MDT3 strips did not reach statistical significance until 72 hours. The effect of one strip reached significance at Day 5 assessment only, whereas the effects of the higher doses remained statistically significant through Day 7. The effect of MDT3, as expressed as fold increase in withdrawal threshold versus Sham, was greatest at 72 hours, before withdrawal threshold began to trend upward in the Sham group (beginning of model resolution). At 72 hours, the mean withdrawal threshold was 3.9, 14.6, 36.8, and 54.8\% of baseline in Sham, one MDT3, two MDT3, and three MDT3, respectively (Figure 2). Daily injections of bupivacaine $(30 \mathrm{mg}$ ) resulted in near-complete reversal of tactile allodynia 1 hour postinjection $(100 \%$ of baseline at 72 hours), whereas similar daily injections of clonidine (up to $225 \mu \mathrm{g} /$ day) had a modest but significant effect (34\% of baseline at 72 hours, Figure 2 ).

No toxicologically adverse effects were recorded during the 1 week postsurgical observation period. However, there was evidence of sedation as measured by a reduction in spontaneous motor activity, particularly in the clonidine injection group (Table 2). Subincisional injection of clonidine solution $(225 \mu \mathrm{g} /$ day $)$ was associated with $41.1,26.0$, and $21.9 \%$ reductions in the mean number of cells breached compared to baseline at 4,24 , and 48 hours, respectively. Two MDT3 was associated with a $26.9 \%$ reduction SMA at 4 hours and a $19.2 \%$ reduction at 24 hours, with complete recovery by 48 hours. Effects in other treatment groups failed to reach statistical significance (Table 2).

Table 2 Spontaneous locomotor activity

\begin{tabular}{|c|c|c|c|c|c|}
\hline \multirow[t]{2}{*}{ Treatment } & \multirow[t]{2}{*}{$n$} & \multicolumn{4}{|c|}{ Time point } \\
\hline & & Baseline & 4 hours & 24 hours & 48 hours \\
\hline Sham & 8 & $99 \pm 7$ & $106 \pm 10$ & $96 \pm 9$ & $88 \pm 9$ \\
\hline Placebo & 8 & $64 \pm 10$ & $59 \pm 10$ & $74 \pm 10$ & $84 \pm 11$ \\
\hline I MDT3 & 8 & $75 \pm 11$ & $82 \pm 11$ & $90 \pm 17$ & $87 \pm 18$ \\
\hline 2 MDT3 & 8 & $78 \pm 16$ & $57 \pm 10 * * *$ & $63 \pm 7^{* *}$ & $79 \pm 11$ \\
\hline 3 MDT3 & 8 & $61 \pm 8$ & $64 \pm 9$ & $72 \pm 8$ & $76 \pm 8$ \\
\hline Bupivacaine (30 mg) & 8 & $81 \pm 5$ & $91 \pm 10$ & $82 \pm 7$ & $87 \pm 11$ \\
\hline Clonidine $(225 \mu g)$ & 4 & $73 \pm 9$ & $43 \pm 4 * * *$ & $54 \pm 6 * * *$ & $57 \pm 4 * *$ \\
\hline
\end{tabular}

Notes: Data represent mean number of cell breaches in 2 minutes per group \pm SD. Data were analyzed by repeated measures ANOVA, followed by Dunnett's multiple comparison post hoc, comparing activity at each postoperative assessment with baseline within each treatment group. Both the time $(P=0.007, F=5.91)$ and treatment $(P<0.000 \mathrm{I}, F=58.17)$ main effects reached significance, as did the interaction between main effects $(P<0.000 \mathrm{I}, F=6.47)$. ${ }^{* * P}<0.0 \mathrm{I}$ for reduction versus baseline. ${ }^{* * *} P<0.00 \mathrm{I}$ for reduction versus baseline.
To further assess the dose-response effects of clonidine strips on peri-incisional tactile allodynia, a cumulative withdrawal threshold from 4 hours to 7 days postimplantation was calculated (Figure 3 ). The mean cumulative withdrawal threshold was 2.3 times higher in Group 3 (1× MDT3) compared to Group 1 (Sham), although this narrowly failed to reach statistical significance (Figure 3). The mean cumulative withdrawal threshold was 3.1 times higher than Sham in Group $4(2 \times$ MDT3, $P<0.01)$ and 3.9 times higher than Sham in Group $5(3 \times$ MDT3, $P<0.001)$. Cumulative withdrawal threshold was over five times higher than Sham in the bupivacaine group $(P<0.0001)$. The effect of daily clonidine solution on this summed end point did not reach statistical significance (Figure 3). There was a statistically significant correlation between dose of MDT3 and cumulative withdrawal threshold from 4 hours to 7 days (Figure 4).

The mean rate of drug release was highest during first 4 hours, averaging $18.1 \pm 0.32 \mu \mathrm{g} /$ hour/strip (Figure 5). This fell to $5.4 \pm 0.32 \mu \mathrm{g} / \mathrm{hour} / \mathrm{strip}$ between 4 and 24 hours and to $<5 \mu \mathrm{g} /$ hour/strip thereafter. Drug release per day was $<150 \mu \mathrm{g}$ after 24 hours (the typical dose in orthopedic postoperative pain studies) (Figure 5). ${ }^{15-17}$ Expressed as HED, the rate of drug release falls below the recommended starting dose for epidural administration ( $30 \mu \mathrm{g} /$ hour in man) by 6 hours (per biphasic nonlinear regression model, Figure 5).

\section{Discussion}

These data suggest that peri-incisional application of clonidine in a sustained-release depot formulation may be a viable alternative to amide anesthetics for the treatment of the acute nociceptive sensitization component of postoperative pain. A strong dose-response relationship was found from 0.77 to $2.3 \mathrm{mg}$ clonidine $\mathrm{HCl}$ (in the form of $1-3 \mathrm{MDT} 3$ ). The effect of three MDT3 strips in ameliorating peri-incisional tactile allodynia reached statistical significance by the first assessment at 4 hours and remained significant through the end of the study (Day 7). Despite the sustained efficacy, the rate of drug release (expressed as HED) fell below those associated with centrally/systemically mediated analgesia during the first several hours. Since three MDT3 strips were the highest dose tested, the maximally effective dose in this model cannot be concluded from this study. It was noteworthy that the effect of daily sub-incisional bolus injection of $225 \mu \mathrm{g}$ (1.80 mg cumulative dose) was not significant in the cumulative analysis. The modest effect in this group reached significance at 72 hours only. Given that tactile allodynia was assessed 1 hour after the peri-incisional injections and the efficacy of doses in this range reported in the literature, 
this was unexpected. The lack of pharmacokinetics data to confirm expected (high) systemic exposure in this group hinders a definitive interpretation. Nonetheless, the clonidine injection group exhibited the greatest magnitude of systemic sedation, as assessed by spontaneous motor activity. While statistical power was inadequate to detect subtle effects, the injection group trended inferior to the one MDT3 strip group in the cumulative tactile allodynia analysis. This suggests that the polymer depot delivery system may have potency as well as duration of action advantages compared to a bolus solution by better concentrating the drug near local pain generators and slowing systemic absorption and clearance. The greater summed effect on tactile withdrawal threshold combined with evidence of less systemic drug effects in the MDT3 groups compared to the clonidine injection group is consistent with a primarily local (peripheral) mechanism of action.

The analgesic effects of clonidine have historically been considered primarily, if not exclusively, centrally mediated. ${ }^{21}$ Stimulation of $\alpha_{2}$ adrenergic receptors (ARs) in the CNS plays an undisputed role in the pharmacological effects of epidurally administered clonidine. However, accumulating evidence demonstrates distinct, peripherally mediated actions that could contribute to analgesia. Intra-articular or periarticular administration of $150 \mu \mathrm{g}$ clonidine has been shown to provide an analgesic effect following arthroscopic knee $e^{15-17,22}$ and shoulder surgery. ${ }^{18}$ Consistent with a local mechanism of action, subcutaneous administration of the same dose had no effect on postoperative pain or consumption of rescue medication. ${ }^{18,22}$ It is important to note, however, that the contribution of deeper soft tissue pain generators (eg, articular cartilage and ligaments) is expected to result in more severe and durable pain (eg, higher summed pain intensity) compared to an incisional model limited to superficial somatic pain.

There are several limitations to using nonclinical evoked pain behavior data to predict effects on patient-reported "spontaneous" pain. Evoked and spontaneous pain are distinct phenomena, with the latter generally appearing to be more responsive to drugs with a central mechanism of action. ${ }^{23}$ Although behavioral measures of spontaneous pain have been proposed, ${ }^{23}$ there is no validated nonclinical equivalent to common patient-reported outcomes (eg, Numeric Rating Scale or visual analog scale at rest). Furthermore, many “evoked pain" models, including the porcine model described in this article, cannot definitively distinguish a complete afferent pain pathway and response from a nociceptive response that circumvents higher brain centers. While nociception and pain can occur independently, their concordance is likely better in the case of acute tissue injury compared to chronic pain models. In this context, nociception is the peripheral component to evoked pain from applying a stimulus to injured tissue. An antinociceptive effect, therefore, likely has clinical relevance to evoked pain in healing tissue.

The precise mechanism of this local, peripheral analgesic effect of clonidine requires further research. Antiinflammatory effects have been described in clinical and mechanistic nonclinical studies. ${ }^{10-14}$ Anti-inflammatory effects appear to be $\alpha_{2}$ AR dependent and may involve the stimulation of these receptors on macrophages. Clonidine has also been shown to act directly on peripheral nerves in vitro to produce local anesthetic effects, interfering with the activity of voltage-gated $\mathrm{Na}^{+}$channels and, at sufficient doses, can mediate a complete regional nerve blockade. ${ }^{24}$ This anesthetic-like effect appears to be independent of $\alpha_{2}$ $\mathrm{ARs}^{24}$ and, therefore, may not require exposure of nerve endings to drug (ie, clonidine may interfere with pain signaling anywhere along axons). This mechanism may contribute to postoperative analgesia, with lower concentrations than what is required to achieve a complete nerve block blunting the propagation of action potentials by pain fibers (ie, fewer impulses through fiber bundle per unit time or smaller whole nerve action potential amplitude). Stimulation of $\alpha_{2}$ ARs on peripheral nerve endings likely also contributes to analgesia. ${ }^{25}$ While the central analgesic effect of clonidine has been shown to be blocked by an $\alpha_{2}$ AR antagonist, ${ }^{26}$ the relative contribution of $\alpha_{2}$ AR-independent effects to peripherally mediated analgesia is unclear. In vitro studies suggest that the anesthetic-like effects of clonidine require higher concentrations than those associated with alpha-2 AR-dependent effects. ${ }^{23,27}$ A hydrolysis-dependent sustained-release depot may facilitate reaching higher concentrations within axon bundles compared to local injections of clonidine solution.

Although a central component to the analgesic effects of this MDT3 prototype clonidine depot in the pig incisional pain model cannot be excluded with certainty, in vitro drug elution data indicate that the lowest effective dose releases drug at a significantly slower rate compared to the recommended starting dose for epidural clonidine infusion. Moreover, epidural administration of clonidine is associated with CSF concentrations up to 10 times the systemic plasma levels. ${ }^{28}$ Therefore, CSF concentrations at the lowest effective dose are unlikely to have been maintained at levels associated with clinically meaningful central analgesia. The failure of bolus subcutaneous administration of $150 \mu \mathrm{g}$ clonidine $\mathrm{HCl}$ to elicit an analgesic effect in $\operatorname{man}^{18,22}$ further supports the supposition of a localized, peripheral mechanism of action. 
Finally, the failure of peri-incisional daily $225 \mu \mathrm{g}$ boluses of clonidine to significantly affect tactile allodynia 1 hour postadministration is strongly suggestive that the efficacy of MDT3 was not dependent on systemic absorption. The rate of release from MDT3 would have approached the bolus dose during the first hour postadministration only, whereas the magnitude of efficacy expressed as a percent increase over Sham generally peaked at 72 hours.

While the clinical relevance of these findings remains uncertain, the peripheral component of nociception is well conserved across species. It is most plausible that these results would have translational value in the context of superficial somatic pain, particularly nociceptive sensitization associated with a full thickness skin incision. The local efficacy of a clonidine depot for other types of pain that may be associated with surgery, such as deep somatic pain from periosteal nerve endings, visceral pain from thoracic or abdominal cavity procedures, and neuropathic pain from certain spinal procedures, requires further investigation. Further elucidation of the precise local mechanism(s) of action is of immense importance in determining the appropriateness of sustained clonidine delivery for these different types of pain as well as the ideal delivery system for concentrating the drug at the site(s) of action.

\section{Acknowledgment}

The subject matter of this article was conducted as part of a Medtronic-sponsored acute pain research and product development initiative and was included in support of an FDA Investigational New Drug submission. This study was funded by Medtronic, Plc., and was reported to the FDA in support of IND 113755 and NCT02077140.

\section{Disclosure}

The authors report no conflicts of interest in this work.

\section{References}

1. Koh IJ, Kang YG, Chang CB, Do SH, Seong SC, Kim TK. Does periarticular injection have additional pain relieving effects during contemporary multimodal pain control protocols for TKA? A randomised, controlled study. Knee. 2012;19(4):253-259.

2. Lee SK, Lee JW, Choy WS. Is multimodal analgesia as effective as postoperative patient-controlled analgesia following upper extremity surgery? Orthop Traumatol Surg Res. 2013;99(8):895-901.

3. Jo CH, Shin JS, Huh J. Multimodal analgesia for arthroscopic rotator cuff repair: a randomized, placebo-controlled, double-blind trial. Eur J Orthop Surg Traumatol. 2014;24(3):315-322.

4. Rafiq S, Steinbrüchel DA, Wanscher MJ, et al. Multimodal analgesia versus traditional opiate based analgesia after cardiac surgery, a randomized controlled trial. J Cardiothorac Surg. 2014;9:52.

5. Devin CJ, McGirt MJ. Best evidence in multimodal pain management in spine surgery and means of assessing postoperative pain and functional outcomes. J Clin Neurosci. 2015;22(6):930-938.
6. Golf M, Daniels SE, Onel E. A phase 3, randomized, placebo-controlled trial of DepoFoam(R) bupivacaine (extended-release bupivacaine local analgesic) in bunionectomy. Adv Ther. 2011;28(9):776-788.

7. Sommer M, de Rijke JM, van Kleef M, et al. The prevalence of postoperative pain in a sample of 1490 surgical inpatients. Eur J Anaesthesiol. 2008;25(4):267-274.

8. Piper SL, Kramer JD, Kim HT, Feeley BT. Effects of local anesthetics on articular cartilage. Am J Sports Med. 2011;39(10):2245-2253.

9. Karpie JC, Chu CR. Lidocaine exhibits dose- and time-dependent cytotoxic effects on bovine articular chondrocytes in vitro. Am J Sports Med. 2007;35(10):1621-1627.

10. Kim MH, Hahn TH. The effect of clonidine pretreatment on the perioperative proinflammatory cytokines, cortisol, and ACTH responses in patients undergoing total abdominal hysterectomy. Anesth Analg. 2000;90(6):1441-1444.

11. Lavand'homme PM, Eisenach JC. Perioperative administration of the alpha2-adrenoceptor agonist clonidine at the site of nerve injury reduces the development of mechanical hypersensitivity and modulates local cytokine expression. Pain. 2003;105(1-2):247-254.

12. Romero-Sandoval A, Eisenach JC. Clonidine reduces hypersensitivity and alters the balance of pro- and anti-inflammatory leukocytes after local injection at the site of inflammatory neuritis. Brain Behav Immun. 2007;21(5):569-580.

13. Romero-Sandoval EA, McCall C, Eisenach JC. Alpha2-adrenoceptor stimulation transforms immune responses in neuritis and blocks neuritisinduced pain. J Neurosci. 2005;25(39):8988-8994.

14. Lavand'homme PM, Ma W, De Kock M, Eisenach JC. Perineural alpha(2A)-adrenoceptor activation inhibits spinal cord neuroplasticity and tactile allodynia after nerve injury. Anesthesiology. 2002;97(4):972-980.

15. Alagol A, Calpur OU, Usar PS, Turan N, Pamukcu Z. Intraarticular analgesia after arthroscopic knee surgery: comparison of neostigmine, clonidine, tenoxicam, morphine and bupivacaine. Knee Surg Sports Traumatol Arthrosc. 2005;13(8):658-663.

16. Buerkle $\mathrm{H}$, Huge $\mathrm{V}$, Wolfgart $\mathrm{M}$, et al. Intra-articular clonidine analgesia after knee arthroscopy. Eur J Anaesthesiol. 2000;17(5):295-299.

17. Gentili M, Enel D, Szymskiewicz O, Mansour F, Bonnet F. Postoperative analgesia by intraarticular clonidine and neostigmine in patients undergoing knee arthroscopy. Reg Anesth Pain Med. 2001;26(4):342-347.

18. Iskandar H, Benard A, Ruel-Raymond J, Cochard G, Manaud B. The analgesic effect of interscalene block using clonidine as an analgesic for shoulder arthroscopy. Anesth Analg. 2003;96(1):260-262, table of contents.

19. Castel D, Willentz E, Doron O, Brenner O, Meilin S. Characterization of a porcine model of post-operative pain. Eur J Pain. 2014;18(4):496-505.

20. Brennan TJ, Vandermeulen EP, Gebhart GF. Characterization of a rat model of incisional pain. Pain. 1996;64(3):493-501.

21. Duraclon ${ }^{\circledR}$ Product Label Bioniche Pharma. Lake Forest, IL: 2010

22. Gentili M, Juhel A, Bonnet F. Peripheral analgesic effect of intraarticular clonidine. Pain. 1996;64(3):593-596.

23. Murai N, Sekizawa T, Gotoh T, et al. Spontaneous and evoked painassociated behaviors in a rat model of neuropathic pain respond differently to drugs with different mechanisms of action. Pharmacol Biochem Behav. 2016;141:10-17.

24. Leem JW, Choi Y, Han SM, Yoon MJ, Sim JY, Leem SW. Conduction block by clonidine is not mediated by alpha2-adrenergic receptors in rat sciatic nerve fibers. Reg Anesth Pain Med. 2000;25(6):620-625.

25. Nakamura M, Ferreira SH. Peripheral analgesic action of clonidine: mediation by release of endogenous enkephalin-like substances. Eur J Pharmacol. 1988;146(2-3):223-228.

26. Yesilyurt O, Uzbay IT. Agmatine potentiates the analgesic effect of morphine by an alpha(2)-adrenoceptor-mediated mechanism in mice. Neuropsychopharmacology. 2001;25(1):98-103.

27. Wolff M, Heugel P, Hempelmann G, Scholz A, Mühling J, Olschewski A. Clonidine reduces the excitability of spinal dorsal horn neurones. Br J Anaesth. 2007;98(3):353-361.

28. US FDA Center for Evaluation and Research Duraclon $0.1 \mathrm{mg} / \mathrm{mL}$ Injection Approval Package (Fujisawa). 1996. 
The Journal of Pain Research is an international, peer reviewed, open access, online journal that welcomes laboratory and clinical findings in the fields of pain research and the prevention and management of pain. Original research, reviews, symposium reports, hypothesis formation and commentaries are all considered for publication
The manuscript management system is completely online and includes a very quick and fair peer-review system, which is all easy to use. Visit http://www.dovepress.com/testimonials.php to read real quotes from published authors.

Submit your manuscript here: https://www.dovepress.com/journal-of-pain-research-journal 